\title{
SBC2008-XXXXXX
}

\section{FUNCTIONAL MOBILITY TESTING: A NOVEL METHOD TO ESTABLISH HUMAN- SYSTEM INTERFACE DESIGN REQUIREMENTS}

\author{
Scott A. England (1) \\ Elizabeth A. Benson (1) \\ Sudhakar Rajulu (2)
}

\author{
(1)MEI Technologies, Inc. \\ Houston, Texas
}

\author{
(2) Anthropometry \& Biomechanics Facility \\ National Aeronautics and Space Administration \\ Houston, TX
}

\section{INTRODUCTION}

Across all fields of human-system interface design it is vital to posses a sound methodology dictating the constraints on the system based on the capabilities of the human user. These limitations may be based on strength, mobility, dexterity, cognitive ability, etc. and combinations thereof. Data collected in an isolated environment to determine, for example, maximal strength or maximal range of motion would indeed be adequate for establishing not-to-exceed type design limitations, however these restraints on the system may be excessive over what is basally needed. Resources may potentially be saved by having a technique to determine the minimum measurements a system must accommodate.

This paper specifically deals with the creation of a novel methodology for establishing mobility requirements for a new generation of space suit design concepts. Historically, the Space Shuttle and the International Space Station vehicle and space hardware design requirements documents such as the Man-Systems Integration Standards [1] and International Space Station Flight Crew Integration Standard [2] explicitly stated that the designers should strive to provide the maximum joint range of motion capabilities exhibited by a minimally clothed human subject. In the course of developing the Human-Systems Integration Requirements (HSIR) [3] for the new space exploration initiative (Constellation), an effort was made to redefine the mobility requirements in the interest of safety and cost. Systems designed for manned space exploration can receive compounded gains from simplified designs that are both initially less expensive to produce and lighter, thereby, cheaper to launch.

\section{METHODS}

A novel approach to the quantification of required mobility was used in this test. Rather than looking at current suit mobility, which may not provide adequate mobility to perform all functional tasks, or looking at maximum unsuited mobility, which is unrealistic and unnecessary to design into a suit, the new approach focused instead on functional range of motion. Setting design requirements based on the mobility necessary to perform a broad spectrum of functional tasks should save resources, compared to requirements to supply the full range of human mobility while still providing a suit capable of performing all tasks that a suited crewmember is likely to encounter. To this end, kinematic data was collected while 20 healthy subjects (10 male, 10 female) performed an array of functional tasks likely to be required of a suited crewmember at some point in a lunar mission. This functional task list included such actions as walking, crawling, manipulating cargo, rotating a hatch, climbing a ladder, ingressing a recumbent seat and many more. Maximum isolated joint mobility was collected additionally to provide a point of reference.

\section{Data Collection}

All data was collected over a three-month period in the Anthropometry and Biomechanics Facility in Building 15 at Johnson Space Center. Kinematic data was recorded at $200 \mathrm{~Hz}$ with a Vicon 612/SV (Oxford Metrics, Oxford, UK) passive video-based motion analysis system containing 10 cameras for this study. Forty-one retroreflective markers were placed at various points on the subject with at least 3 points per major body segment to enable the calculation of major joint angles. Data were processed through custom written Vicon BodyBuilder models and Matlab programs to populate a spreadsheet for every subject with extreme ranges of motion for every joint during every task. 


\section{Data Analysis}

Once joint angles were calculated for every functional task, maximum ranges of motion (ROM) were extracted for each joint of every subject across all tasks. Modal analyses of the maximum functional mobility data successfully consolidated moderately variable results into a table of single values per motion. The mode was used as it was deemed less vulnerable to outliers than the median or the mean and the mode is uniquely qualified as a statistical method for accommodating different approaches taken to completing a functional task. If, for example, slightly more than half of the subject pool used a large amplitude of some specific joint rotation to complete a task and the remaining subjects completed the task in a different manner that used a very small rotation of the same joint, the mean would report a value in the middle, denying more than half the subjects the required joint mobility to complete the task in their preferred manner. The median would likely report one of the lowest values in the larger group, still not providing many of the subjects with the mobility they utilized to complete the task in their desired fashion. Because Bodybuilder provides angles calculated to six decimal places, the reported joint angles were rounded to the nearest multiple of 5 degrees. This was done so that a mode could be calculated for the data. This rounding was deemed justifiable after an inspection of the data, which suggested that consecutive extreme ranges of motion for a variety of cyclic tasks were separated by no more than 5 degrees. In other words, as a subject completed a cyclic task (hammering, walking or shoveling are examples), they appeared to reach a consistent peak range of motion throughout the test, which generally varied less than 5 degrees.

Since the mode is defined as the most frequently occurring value, it is possible for multiple modes to exist for a given measure. While rare, this problem would prevent a single value from being reported as a design requirement so when multiple modes exist, the value that was closest to the mean of the data was selected.

\section{RESULTS}

Maximum functional mobility was consistently lower than maximum isolated mobility. Exceptions to this trend were noticed for a few specific joint rotations and can generally be explained by the manner in which isolated mobility was collected. For isolated mobility trials, subjects were asked to suspend the investigated limb in a neutral position and then move it through its extreme range of motion through the use of their own musculature. For certain joints however, such as the wrist and ankle, greater ranges of motion may be achieved when significant weight is put behind the motion such as during functional tasks including crawling on hands and knees and climbing a ramp. Despite several exceptions, required functional mobility provided a marked reduction from the maximum isolated mobility of which subjects were capable.

\section{CONCLUSION}

This study suggested that conventional methods for establishing design requirements for human-systems interfaces based on maximal capabilities may be excessive. Additionally, this method provides a valuable means for evaluating systems created from these requirements by comparing the mobility available in a new spacesuit or required to use some new piece of hardware to this newly established archive of functional mobility.

\section{REFERENCES}

1. Man-Systems Integration Standards. Lyndon B. Johnson Space Center, Houston, Texas, October 1995. NASA-STD-3000, Revision B.

2. International Space Station Fight Crew Integration Standard. Lyndon B. Johnson Space Center, Houston, Texas, June 2006

3. Human Systems Integration Requirements. Lyndon B. Johnson Space Center, Houston, Texas, In Press. NASA-CXP70024. 